\title{
Exponential Stability for the Schlögl System by Pyragas Feedback
}

\section{Martin Gugat $^{1}$ (D) . Mariano Mateos ${ }^{2} \cdot$ Fredi Tröltzsch $^{3}$}

Received: 22 July 2019 / Accepted: 24 October 2019 / Published online: 25 February 2020

(C) The Author(s) 2020

\begin{abstract}
The Schlögl system is governed by a nonlinear reaction-diffusion partial differential equation with a cubic nonlinearity. In this paper, feedback laws of Pyragas-type are presented that stabilize the system in a periodic state with a given period and given boundary traces. We consider the system both with boundary feedback laws of Pyragas type and distributed feedback laws of Pyragas and classical type. Stabilization to periodic orbits is important for medical applications that concern Parkinson's disease. The exponential stability of the closed loop system with respect to the $L^{2}$-norm is proved. Numerical examples are provided.
\end{abstract}

Keywords Lyapunov function · Boundary feedback · Robin feedback · Parabolic partial differential equation - Exponential stability $\cdot$ Stabilization of periodic orbits $\cdot$ Periodic operation · Stabilization of desired orbits · Poincaré-Friedrichs inequality · Delay differential equations

Mathematics Subject Classification (2010) 49J20 • 93B52 • 93C20

Dedicated to Volker Mehrmann on the occasion of his 65th birthday.

Martin Gugat

martin.gugat@fau.de

Mariano Mateos

mmateos@uniovi.es

Fredi Tröltzsch

troeltzsch@math.tu-berlin.de

1 Department Mathematik, Friedrich-Alexander-Universität Erlangen-Nürnberg, Cauerstr. 11, 91058 Erlangen, Germany

2 Departmento de Matematicas E.P.I. Gijón Universidad de Oviedo, 33203, Gijón, Spain

3 Institut für Mathematik, Technische Universität Berlin, Str. des 17. Juni 136, 10623 Berlin, Germany 


\section{Introduction}

The Schlögl system introduced in [28] is a model for chemical reactions for non-equilibrium phase transitions that describes the concentration of a substance in dimension 1. In neurology, the same system is known as Nagumo equation (or Newell-Whitehead-Segel equation, see [26, 29]) and models an active pulse transmission through an axon [8, 25]. This system is governed by a parabolic partial differential equation with a cubic nonlinearity that determines three constant equilibrium states $u_{1}<u_{2}<u_{3}$, where $u_{2}$ is unstable.

The Schögl model serves as a simplified model problem for more complicated equations such as the bidomain system in heart medicine, cf. [19]. Here, the goal of stabilization is to extinguish undesired spiral waves as fast as possible and hereafter to control the system to a desired state. However, there are similarities between these models and it is therefore reasonable to consider related questions for the Schögl system. A finite-dimensional dynamic compensator for the Schlögl model is designed in [3]. Extensive Monte Carlo calculations of Schlögl's model for chemical reactions are presented in [15].

The control functions can act in the domain (distributed control) or on its boundary. In this paper, the problem of feedback stabilization towards periodic orbits is studied. For instance, the construction of Lasers with a desired oscillation behavior is an interesting issue for feedback stabilization.

A possible application of the boundary stabilization of time-periodic orbits in the Nagumo system is the boundary stabilization of a periodic pulse transmission. Another important motivation is the development of new treatments for Parkinson's disease. In [17], linear combinations of a fixed number of Dirac measures are used in experiments that are related to the treatment of Parkinson's disease. There are also interesting applications in Theoretical Physics such as the control of cluster synchronization in [20] and time-delayed stabilization of solutions to nonlinear differential equations in [9]. The existence of periodic solutions of nonlinear parabolic equations is studied in [27]. Stabilization towards desired periodic orbits is also considered in [1] for a pendulum system and in [23, 24] for discrete time chaotic systems. Optimal control problem for systems governed by the stochastic FitzHugh-Nagumo equation with a Gaussian noise are studied in [2]. Similar optimal control problems with recovery variable are considered in [9].

In [16] we have studied the boundary stabilization to given desired orbits with linear Robin-feedback laws. In this new paper, we concentrate on the exponential stabilization towards a periodic orbit with feedback laws of Pyragas type. In particular, we are interested in periodic orbits with desired oscillations. We consider both boundary and distributed feedback control. In our analysis we study the exponential decay of the Lyapunov function

$$
V(t)=\frac{1}{2} \int_{0}^{L}(u(t, x)-u(t-T, x))^{2} d x,
$$

where $u(t, x)$ denotes the system state at time $t$ and position $x$ and $T$ stands for a desired period length.

To show that the system is exponentially stable, we verify that $V$ is a strict Lyapunov function. The construction of strict Lyapunov functions for semilinear parabolic partial differential equations has also been studied in [22]. In [22], it is assumed that the feedback is space-periodic or the boundary conditions are chosen in such a way that the product of the state and the normal derivative vanishes at the boundary. This assumption implies that the boundary terms occurring after partial integration in the time derivative of the Lyapunov function become nonpositive. For the state feedback laws that are presented in this paper, this assumption holds. But due to the time delay in the Pyragas feedback term, the 
analysis from [22] is not directly applicable. Therefore a different approach is used in the analysis: For the boundary control, a Poincaré-Friedrichs inequality is used to show that the Lyapunov function is strict.

The Schlögl system has the interesting property that it allows traveling wave solutions (i.e., uniformly translating solutions moving with a constant velocity) which have the shape of the hyperbolic tangent (see [18]). The traveling wave solutions connect the two stable constant stationary states $u_{1}$ and $u_{3}$. The problem to steer associated wave fronts to rest by distributed optimal control methods with finite time horizon was considered in [5] for the Schlögl model and in [6] for the FitzHugh-Nagumo system, where spiral waves occur. In the present paper, we propose control laws that stabilize the system exponentially fast to a periodic orbit.

In this paper, a spatially 1-d system of length $L$ is studied. In the reaction-diffusion equation, the diffusion coefficient is normalized to 1 . The parameter $\rho$ determines the size of the reaction term.

In particular, we are interested in answering the following question: Let a state function $u$ be given that exhibits a stable oscillatory behavior in a fixed bounded time horizon $[0, T]$. Will this function approach a periodic and oscillating orbit as $t \rightarrow \infty$ ?

This paper has the following structure: In Section 2, a system is studied where the Pyragas terms appear in the boundary conditions. The model is defined and a result about the well-posedness is given. We give conditions that guarantee that the system converges to a periodic orbit exponentially fast.

In Section 3, the result about feedback stabilization where the Pyragas terms appear as distributed controls is presented. The feedback gain can be chosen in such a way that the system converges exponentially fast to a periodic state with the desired period $T$. Similar results are shown for distributed feedback laws of classical type.

For the boundary feedback law, the Pyragas terms acts through the boundary conditions. If the desired $T$-periodic orbit is determined uniquely by these boundary traces, under appropriate assumptions the system states converges exponentially fast to the desired $T$-periodic orbit.

For the distributed feedback law, the Pyragas term acts directly in the partial differential equation (pde, for short) whereas in the boundary conditions, desired Neumann boundary traces for the $T$-periodic orbit are prescribed. Numerical experiments that illustrate the behavior of the system are presented in Section 5. Section 6 contains conclusions.

\section{The Schlögl Model with Pyragas Boundary Feedback Control}

\subsection{Definition of the Schlögl Model}

Let real numbers $u_{1} \leq u_{2} \leq u_{3}$ be given and define the polynomial

$$
R(u)=\left(u-u_{1}\right)\left(u-u_{2}\right)\left(u-u_{3}\right) .
$$

Due to its definition, $R$ has the property

$$
m_{R}=\inf _{u \in(-\infty, \infty)} R^{\prime}(u)>-\infty
$$

hence the derivative of $R$ is bounded from below. The infimum $m_{R} \leq 0$ is attained at the point $\left(u_{1}+u_{2}+u_{3}\right) / 3$. 
The system that is considered in this paper is governed by the semilinear parabolic partial differential equation

$$
u_{t}=u_{x x}-\rho R(u)
$$

with a constant $\rho>0$ complemented by appropriate initial and boundary conditions. In the reaction diffusion equation (3), the diffusion coefficient is equal to 1 and the constant $\rho$ determines the size of the reaction term. If $\rho$ equals zero, the reaction term vanishes and the partial differential equation (3) models a pure linear diffusion process.

Let the length $L>0$, a desired period $T>0$, and a feedback gain $\gamma \in \mathbb{R}$ be given. We consider two versions of feedback laws.

First, we consider distributed feedback of the form

$$
u_{t}=u_{x x}-\rho R(u)+\gamma\left(u-u^{\text {desi }}\right),
$$

where $u^{\text {desi }}$ is a desired state function.

Second, we define also a boundary feedback law. For the stabilization of (4), for $(t, x) \in$ $(0, \infty) \times(0, L)$, with some real constant $C \geq \frac{1}{2 L}$, we consider the Pyragas-Robin boundary conditions

$$
\begin{aligned}
u_{x}(t, 0) & =C[u(t, 0)-u(t-T, 0)]+u_{x}(t-T, 0), \\
u_{x}(t, L) & =-C[u(t, L)-u(t-T, L)]+u_{x}(t-T, L) .
\end{aligned}
$$

In order to start the system, for $t \in(-T, 0)$ we prescribe some sufficiently regular initial state. With the feedback laws (5) and (6), if

$$
2 \rho\left|m_{R}\right|+2 \gamma<\frac{1}{L^{2}}
$$

(see (10) in Theorem 2) the Lyapunov function $V(t)$ defined in (1) decays exponentially.

\subsection{Existence and Uniqueness of the Solutions}

In [5], the well-posedness of the system governed by (3) is studied for homogeneous Neumann boundary conditions. It is shown that for initial data in $L^{\infty}(0, L)$, the system has a unique weak solution that is continuous for $t>0$. If the initial state is continuous, the solution of the system is continuous for all times. In the associated theorem below, we use the standard Sobolev space

$$
W(0, T)=L^{2}\left(0, T, H^{1}(0, L)\right) \cap H^{1}\left(0, T ; H^{1}(0, L)^{\prime}\right)
$$

and write $Q_{T}=(0, T) \times(0, L)$.

Let us first consider the following semilinear parabolic problem with inhomogeneous Robin boundary conditions but without time delay:

$$
\begin{aligned}
u_{t}(t, x)-\rho u_{x x}(t, x)+R(u(t, x)) & =f(t, x) & & \text { in } Q_{T}, \\
u_{x}(t, 0)-C u(t, 0) & =g_{1}(t) & & \text { in }(0, T), \\
u_{x}(t, L)+C u(t, L) & =g_{2}(t) & & \text { in }(0, T), \\
u(0, x) & =u_{0}(x) & & \text { in }(0, L) .
\end{aligned}
$$

Lemma 1 Suppose that it holds $K>0$ and $u_{1} \leq u_{2} \leq u_{3}$. Then, for all $f \in L^{2}\left(Q_{T}\right)$, $u_{0} \in L^{\infty}(0, L), g_{i} \in L^{p}(0, T), i=1,2, p>2$, the parabolic initial-boundary value problem (7) has a unique solution $u$ in

$$
L^{\infty}\left(Q_{T}\right) \cap W(0, T) \cap C((0, T] \times[0, L]) .
$$


If $u_{0} \in C[0,1]$, then $u$ is also continuous on $[0, T] \times[0, L]$.

Proof We apply the standard substitution $u(t, x)=e^{\lambda t} v(t, x)$ and obtain the problem

$$
\begin{aligned}
v_{t}(t, x)-\rho v_{x x}(t, x)+\lambda v(t, x)+e^{-\lambda t} R\left(e^{\lambda t} v(t, x)\right) & =e^{-\lambda t} f(t, x) & & \text { in } Q_{T}, \\
v_{x}(t, 0)-C v(t, 0) & =e^{-\lambda t} g_{1}(t) & & \text { in }(0, T), \\
v_{x}(t, L)+C v(t, L) & =e^{-\lambda t} g_{2}(t) & & \text { in }(0, T), \\
u(0, x) & =u_{0}(x) & & \text { in }(0, L) .
\end{aligned}
$$

If $\lambda$ is taken sufficiently large, then the function $d: v \mapsto \lambda v+e^{-\lambda t} R\left(e^{\lambda t} v\right)$ is monotone nondecreasing. Since $C$ is non-negative, the same holds for the function $b: v \mapsto C v$. Therefore, the problem fits to a general semilinear parabolic problem with monotone nonlinearities that is discussed in [32, Theorem 5.5]. This theorem ensures the existence of a unique bounded solution $v$ in $W(0, T)$ with the claimed regularity properties.

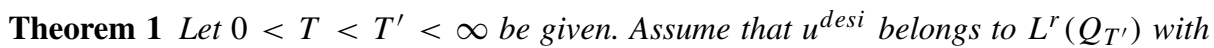
some $r>3 / 2$ and $u_{0} \in C([-T, 0] \times[0, L])$ possesses the partial (one-sided) derivatives $\left(u_{0}\right)_{x}(t, 0):=\left(u_{0}\right)_{x}(t,+0)$ and $\left(u_{0}\right)_{x}(t, L)=\left(u_{0}\right)_{x}(t,-L)$ for all $t \in[-T, 0]$. Let the functions $t \mapsto\left(u_{0}\right)_{x}(t, 0)$ and $t \mapsto\left(u_{0}\right)_{x}(t, L)$ belong to $L^{p}(-T, 0)$ with some $p>2$. Then the parabolic problem with delay $T \in\left(0, T^{\prime}\right]$,

$$
\begin{aligned}
u_{t}-u_{x x}+\rho R(u) & =\gamma\left(u-u^{\text {desi }}\right) & & \text { in } Q_{T^{\prime}}, \\
u(t, x) & =u_{0}(t, x) & & \text { in }(-T, 0) \times(0, L), \\
u_{x}(t, 0)-C u(t, 0) & =u_{x}(t-T, 0)-C u(t-T, 0) & & \text { in }\left(0, T^{\prime}\right), \\
u_{x}(t, L)+C u(t, L) & =u_{x}(t-T, L)+C u(t-T, L) & & \text { in }\left(0, T^{\prime}\right)
\end{aligned}
$$

has a unique solution $u$ such that $\left.u\right|_{\left[0, T^{\prime}\right]} \in W\left(0, T^{\prime}\right) \cap C\left(\left[0, T^{\prime}\right] \times[0, L]\right)$.

Proof We apply the step method that is a classical tool for proving existence and uniqueness of solutions to delay equations. First, we solve the problem on the time interval $[0, T]$. Here, we have $u(t-T, x)=u_{0}(t-T, x)$, hence the problem is one with given Robin boundary data

$$
g_{1}(t)=\left(u_{0}\right)_{x}(t-T, 0)-C u_{0}(t-T, 0), \quad g_{2}(t)=\left(u_{0}\right)_{x}(t-T, L)+C u_{0}(t-T, L)
$$

that fits in (7). Thanks to the assumptions imposed on $u_{0}$, the functions $g_{1}$ and $g_{2}$ belong to $L^{p}(0, T)$ with $p>2$. Therefore and since $u^{\text {desi }}$ belongs to $L^{r}\left(Q_{T^{\prime}}\right)$, Lemma 1 ensures existence and uniqueness of $u$ in $W(0, T) \cap C\left(\overline{Q_{T}}\right)$.

Now we extend the solution $u$ to $\left[T, \min \left\{2 T, T^{\prime}\right\}\right]$. Without stronger assumptions on the regularity of $g_{1}, g_{2}$, and $u_{0}$, we cannot expect that $u_{x}(t-T, 0)$ and $u_{x}(t-T, L)$ exist as functions on $[T, 2 T]$. However, from the boundary conditions we know that $u_{x}(t, 0)-$ $C u(t, 0)=g_{1}(t)$ and $u_{x}(t, L)+C u(t, L)=g_{2}(t)$ exist as real functions in $L^{p}(0, T)$. Therefore, on $[T, 2 T]$ the functions $u_{x}(t-T, 0)-C u(t-T, 0)=g_{1}(t-T)$ and $u_{x}(t-$ $T, L)+C u(t-T, L)=g_{2}(t-T)$ belong to $L^{p}(T, 2 T)$.

In this way, on $\left[T, \min \left\{2 T, T^{\prime}\right\}\right]$ the boundary conditions of (8) can be re-written as

$$
\begin{aligned}
u_{x}(t, 0)-C u(t, 0) & =g_{1}(t-T), \\
u_{x}(t, L)+C u(t, L) & =g_{2}(t-T),
\end{aligned}
$$

$T \leq t \leq \min \left\{2 T, T^{\prime}\right\}$

Moreover, the new initial function $x \mapsto u(T, x)$ is continuous on $[0, L]$, again thanks to Lemma 1 . Therefore, we can apply Lemma 1 again, now on $\left[T, \min \left\{2 T, T^{\prime}\right\}\right]$, to obtain 
existence and uniqueness of $u$ until $\min \left\{2 T, T^{\prime}\right\}$. Repeating this procedure again, we finally can extend the solution up to $T^{\prime}$.

Remark 1 As we have pointed out in the preceding proof, we cannot guarantee that $u_{x}(t, 0)$ and $u_{x}(t, L)$ exist as measurable functions. We resolved this obstacle by using the boundary conditions

$$
\begin{aligned}
u_{x}(t, 0)-C u(t, 0) & =g_{1}(t-(k-1) T), \\
u_{x}(t, L)+C u(t, L) & =g_{2}(t-(k-1) T),
\end{aligned}
$$

on $[(k-1) T, k T], k=1,2, \ldots$. This trick avoids the explicit use of $u_{x}(t-T, 0)$ and $u_{x}(t-T, L)$ in the right-hand side of the boundary conditions. By results on maximal parabolic regularity of [21, Theorems 5.1.17 and 5.1.20], we are able to show on [0,T] the existence of the solution $u$ in $C^{1+\alpha / 2,2+\alpha}\left(\overline{Q_{T}}\right)$ with some $0<\alpha<1$, provided that $u_{0}$ and $u^{\text {desi }}$ have higher regularity. Then the functions $t \mapsto u_{x}(t, 0)$ and $t \mapsto u_{x}(t, L)$ belong to $C^{(1+\alpha) / 2}[0, T]$. For the definition of these spaces, we refer to [21]. Analogously, we can proceed in later time intervals.

\subsection{Exponential Stability}

In this section, we present our main result about the exponential stability in the $L^{2}$-sense of our system with Pyragas boundary control. A boundary feedback law is constructed that stabilizes the system around a given desired $T$-periodic state $u^{d e s i}$.

An essential tool in the analysis is the 1-d POINCARÉ-FRIEDRICHS inequality (see also [32] for the general case) in the following form: let $L>0$ be given. For all $u \in H^{1}(0, L)$, the following inequality holds:

$$
\int_{0}^{L} u^{2}(x) d x \leq L\left[u(0)^{2}+u(L)^{2}\right]+2 L^{2} \int_{0}^{L}\left(\partial_{x} u(x)\right)^{2} d x .
$$

Now the stabilization result for periodic orbits is given. In what follows, we use the notation $Q_{2 T}=(0,2 T) \times(0, L)$.

Theorem 2 (Exponential stability) Let a period $T>0$ and a $T$-periodic state $u^{\text {desi }} \in$ $H^{2}\left(Q_{2 T}\right)$ be given. Assume that $L>0$ is sufficiently small in the sense that

$$
\frac{1}{L^{2}}>2 \rho\left|m_{R}\right|+2 \gamma \text {. }
$$

Let a feedback parameter $C \geq \frac{1}{2 L}$ and an initial state $u_{0} \in C^{1}([-T, 0] \times[0, L])$ be given. Then the solution $u$ of (4) subject to the initial condition

$$
u(t, x)=u_{0}(t, x)
$$

for $t \in(-T, 0)$ and $x \in(0, L)$ and to the boundary conditions

$$
\begin{aligned}
& u_{x}(t, 0)=C(u(t, 0)-u(t-T, 0))+u_{x}(t-T, 0), \\
& u_{x}(t, L)=-C(u(t, L)-u(t-T, L))+u_{x}(t-T, L)
\end{aligned}
$$

for $t \geq 0$ becomes $T$-periodic exponentially fast in the following sense: With

$$
\mu=\frac{1}{L^{2}}-2 \rho\left|m_{R}\right|-2 \gamma,
$$


the inequality

$$
\int_{0}^{L}(u(t, x)-u(t-T, x))^{2} d x \leq \exp (-\mu(t-T)) \int_{0}^{L}\left(u(T, x)-u_{0}(0, x)\right)^{2} d x
$$

holds for all $t \geq T$.

There exist a $T$-periodic function $u^{*} \in L^{2}\left(Q_{2 T}\right)$ and a constant $P_{0}>0$ such that for all $t \geq 0$ we have $u^{*}(t, \cdot) \in L^{2}(0, L)$ and the inequality

$$
\int_{0}^{L}\left(u(t, x)-u^{*}(t, x)\right)^{2} d x \leq P_{0} \exp (-\mu t)
$$

is satisfied for all $t \geq T$.

Proof By Theorem 1 the state $u$ is continuous. Hence, $V$ is well-defined by (1). The definition of $V$ implies that $V(t) \geq 0$. For $t \geq T$, we can write (4) in the form

$$
u_{t}(\cdot-T, \cdot)=u_{x x}(\cdot-T, \cdot)-\rho R(u(\cdot-T, \cdot))+\gamma\left(u(\cdot-T, \cdot)-u^{d e s i}\right) .
$$

Hence, the pde (4) implies that for all $t \geq T$ we have

$$
\begin{aligned}
& u_{t}(t, x)-u x_{t}(t-T, x)-u_{x x}(t, x)+u_{x x}(t-T, x) \\
& =-\rho(R(u(t, x))-R(u(t-T, x))+\gamma(u(t, x)-u(t-T, x))
\end{aligned}
$$

in the sense of the solution of (4) that is given in Theorem 1. Notice that the term with $u^{\text {desi }}$ cancels out by subtracting the pdes for $u(t, x)$ and $u(t-T, x)$. We multiply (13) by $[u(t, x)-u(t-T, x)]$ and integrate to obtain for $t_{0}>T$

$$
\begin{aligned}
& \int_{T}^{t_{0}} \int_{0}^{L}[u(t, x)-u(t-T, x)]\left[\left[u_{t}(t, x)-u_{t}(t-T, x)\right]-\left[u_{x x}(t, x)-u_{x x}(t-T, x)\right]\right] d x d t \\
& =\int_{T}^{t_{0}} \int_{0}^{L}-\rho[u(t, x)-u(t-T, x)](R(u(t, x))-R(u(t-T, x)))+\gamma[u(t, x)-u(t-T, x)]^{2} d x d t .
\end{aligned}
$$

For initial data $u_{0}$ in $H^{2}((-T, 0) \times(0, L))$, integration by parts yields

$$
\begin{aligned}
& \left.\int_{0}^{L} \frac{1}{2}[u(t, x)-u(t-T, x)]^{2}\right|_{t=T} ^{t_{0}} d x+\int_{T}^{t_{0}} \int_{0}^{L}\left[u_{x}(t, x)-u_{x}(t-T, x)\right]^{2} d x d t \\
& =\left.\int_{T}^{t_{0}}[u(t, x)-u(t-T, x)]\left[u_{x}(t, x)-u_{x}(t-T, x)\right]\right|_{x=0} ^{L} d t \\
& +\int_{T}^{t_{0}} \int_{0}^{L}-\rho[u(t, x)-u(t-T, x)](R(u(t, x))-R(u(t-T, x)))+\gamma[u(t, x)-u(t-T, x)]^{2} d x d t .
\end{aligned}
$$

Due to (2), for all $v_{1}, v_{2} \in(-\infty, \infty)$ it holds

$$
\left(v_{2}-v_{1}\right)\left(R\left(v_{2}\right)-R\left(v_{1}\right)\right) \geq\left(v_{2}-v_{1}\right)^{2} m_{R} .
$$

The definition of $V$ and the boundary conditions imply

$$
\begin{aligned}
& V\left(t_{0}\right)-V(T)+\int_{T}^{t_{0}} \int_{0}^{L}\left[u_{x}(t, x)-u_{x}(t-T, x)\right]^{2} d x d t \\
& \leq-\int_{T}^{t_{0}} C[u(t, L)-u(t-T, L)]^{2}+C[u(t, 0)-u(t-T, 0)]^{2} d t \\
& \quad+\int_{T}^{t_{0}} \int_{0}^{L}\left(-\rho m_{R}+\gamma\right)[u(t, x)-u(t-T, x)]^{2} d x d t .
\end{aligned}
$$


Thanks to $C \geq \frac{1}{2 L}$, due to the Poincaré-Friedrichs inequality (9) this implies

$$
V\left(t_{0}\right)-V(T) \leq \int_{T}^{t_{0}} \int_{0}^{L}\left(-\frac{1}{2 L^{2}}-\rho m_{R}+\gamma\right)[u(t, x)-u(t-T, x)]^{2} d x d t .
$$

Now (10) implies $V\left(t_{0}\right) \leq V(T)$. In fact, we have $0 \leq V\left(t_{0}\right) \leq V(T)-\int_{T}^{t_{0}} \mu V(\tau) d \tau$. Now Gronwall's lemma implies the inequality

$$
V\left(t_{0}\right) \leq V(T) \exp \left(-\mu\left(t_{0}-T\right)\right) .
$$

Since $H^{2}((-T, 0) \times(0, L))$ is dense in $L^{\infty}((-T, 0) \times(0, L))$, the same estimate remains true (by continuous extension) for any initial state $u_{0}$ in $L^{\infty}(0, L)$. Thus $V$ is a strict Lyapunov function and the assertion (11) follows. (Similar classical Lyapunov analysis can be found for example in [10] or [11]).

Inequality (11) implies that for all $t \in(0, T)$, the sequence $(u(t+j T, \cdot))_{j=1}^{\infty}$ is a Cauchy sequence in $L^{2}(0, L)$. This can be seen as follows. Define

$$
C_{0}=\sqrt{\int_{0}^{L}\left(u(T, x)-u_{0}(x)\right)^{2} d x} .
$$

Then for all $j \in\{1,2,3, \ldots\}$ we have

$$
\sqrt{\int_{0}^{L}(u(t+j T, x)-u(t+(j-1) T, x))^{2} d x} \leq C_{0} \exp \left(-\frac{\mu}{2}(t+(j-1) T)\right) .
$$

We use the notation $\sqrt{\int_{0}^{L}(f(x))^{2} d x}=\|f\|_{L^{2}(0, L)}$.

For all $k \in\{0,1,2, \ldots\}$ the triangle inequality implies

$$
\begin{aligned}
& \|u(t+(j+k) T, \cdot)-u(t+(j-1) T, \cdot)\|_{L^{2}(0, L)} \\
& \leq \sum_{l=0}^{k}\|u(t+(l+j) T, \cdot)-u(t+(l+j-1) T, \cdot)\|_{L^{2}(0, L)} \\
& \leq C_{0} \sum_{l=0}^{k} \exp \left(-\frac{\mu}{2}(t+(l+j-1) T)\right) \\
& \leq C_{0} \frac{\exp \left(-\frac{\mu}{2}(t+(j-1) T)\right)}{1-\exp \left(-\frac{\mu}{2} T\right)} .
\end{aligned}
$$

Hence, for all $t \in(0, T)$, the sequence $(u(t+j T, \cdot))_{j=1}^{\infty}$ converges in $L^{2}(0, L)$. There exist limit functions $u^{*}(t, \cdot) \in L^{2}(0, L)$ such that, for all $t \in(0, T)$,

$$
\lim _{j \rightarrow \infty} \int_{0}^{L}\left(u(t+j T, x)-u^{*}(t, x)\right)^{2} d x=0 .
$$

Then (11) implies that $u_{*}(t, \cdot)$ generates a $T$-periodic orbit. More precisely, we have

$$
\left\|u^{*}(t, \cdot)-u(t+(j-1) T, \cdot)\right\|_{L^{2}(0, L)} \leq C_{0} \frac{\exp \left(-\frac{\mu}{2}(t+(j-1) T)\right)}{1-\exp \left(-\frac{\mu}{2} T\right)}
$$

which implies (12).

Remark 2 Note that $\gamma<0$ can always be chosen such that (10) holds. 
Example 1 Let $u_{1}=-1, u_{2}=0, u_{3}=\pi$. Then we have $R(u)=\left(u^{2}+u\right)(u-\pi)$ and

$$
m_{R}=-\frac{\pi^{2}+\pi+1}{3}>-5 .
$$

Let $L=1$ and $C=\frac{1}{2}$; the constants $\rho$ and $\gamma$ will be specified below. Consider the desired state $u^{\text {desi }}(t, x)=(\sin (t)+\sin (2 t))^{3} \sin (\pi x)$ which is $T$-periodic with $T=2 \pi$. The corresponding Robin-Pyragas-feedback is

$$
\begin{aligned}
& u_{x}(t, 0)=u_{x}(t-T, 0)+\frac{1}{2}[u(t, 0)-u(t-T, 0)], \\
& u_{x}(t, L)=u_{x}(t-T, L)-\frac{1}{2}[u(t, L)-u(t-T, L)] .
\end{aligned}
$$

- With the choice $\rho=\frac{1}{10}$ and $\gamma=0$, (10) holds. Theorem 2 is applicable and implies the convergence to a $T$-periodic state.

- Consider now $\rho=1$. Then for $\gamma \geq 0$, the inequality (10) is not satisfied. Hence in this case, Theorem 2 is not applicable.

- For $\rho=1$ and $\gamma<-\frac{9}{2}$, the inequality (10) is satisfied and Theorem 2 is applicable.

Remark 3 The feedback strategies of this section can generate timely constant periodic functions, because the standard solutions of the Schlögl model without time delay are constant. In Sections 3 and 4, we investigate the stabilization of the Schlögl model with different stabilization strategies, that are able to generate non-constant periodic limit functions of a desired period.

\subsection{Another Application: A Parabolic Model for Gas Pipeline Flow}

In this section we consider another application of the presented boundary feedback law, namely the flow of ideal gas through a horizontal pipe of length $L>0$. Let numbers $\alpha>0$, $q_{0}>0$ be given. Let $P$ denote the pressure and $q_{0}+q$ the flow rate, where we assume that $|q| / q_{0}$ is sufficiently small. The number $c>0$ denotes the sound speed in the gas. The following system of partial differential equations can be used as a model for the flow for $x \in[0, L]$ and $t>0$ :

$$
P_{t}+c^{2} q_{x}=0, \quad P_{x}=-\alpha \frac{q_{0}^{2}+2 q_{0} q}{P}
$$

(see $[4,12,31])$. From the second equation we obtain $q_{x}=-\frac{1}{2 \alpha q_{0}}\left(\frac{1}{2} P^{2}\right)_{x x}$. In order to obtain a single partial differential equation we insert this in the first equation. This yields

$$
P_{t}=-\frac{c^{2}}{2 \alpha q_{0}}\left(\frac{1}{2} P^{2}\right)_{x x} .
$$

Let $p_{0}(x)>0$ denote a function such that $\left(\frac{1}{2} p_{0}^{2}\right)_{x}=-\alpha q_{0}^{2}$. We introduce a new variable $p$ by $P=p_{0}+p$. If we neglect the lower order terms with $p_{x}, p_{x}^{2}$ we obtain $p_{t}=-\frac{c^{2}}{2 \alpha q_{0}}\left(p_{0}+\right.$ p) $p_{x x}$. By neglecting the quadratic term this yields the linear model

$$
p_{t}=-\frac{c^{2}}{2 \alpha q_{0}} p_{0} p_{x x}
$$

which has the same form as the Schlögl model with zero reaction term, that is $\rho=0$. So we can apply the methods for boundary stabilization from Section 2. This is of interest for the 
operation of gas pipelines since often, the customer demand has a periodic structure (see [14]). If the variations of the demand curves are sufficiently small, that is if the gas flow remains close to the stationary state $\left(q_{0}, p_{0}(x)\right)$, they can be modeled with (15).

\section{The Schlögl Model for Distributed Pyragas Control}

\subsection{Definition of the Model}

Again we introduce a given desired $T$-periodic measurable state $u^{\text {desi }} \in L^{\infty}\left(Q_{2 T}\right)$,

$$
u^{\text {desi }}=u^{\text {desi }}(t, x)
$$

that has well-defined $T$-periodic Neumann traces $u_{x}^{\text {desi }}(\cdot, 0) \in L^{p}(0, T)$ and $u_{x}^{\text {desi }}(\cdot, L) \in L^{p}(0, T), p>2$. We extend $u^{\text {desi }}$ to a $T$-periodic function on $[0, \infty) \times[0, L]$.

Moreover, we assume that an initial state $u_{0} \in C\left([-T, 0], L^{\infty}(0, L)\right)$ is given. Let a real number

$$
\kappa>0
$$

be given. For $(t, x) \in[0, \infty) \times(0, L)$, our system state is defined as the solution of the initial-boundary value problem

(S) :

$$
\begin{aligned}
u_{t}(t, x)= & u_{x x}(t, x)-u_{x x}(t-T, x) \\
& -\rho[R(u(t, x))-R(u(t-T, x))]+u_{t}(t-T, x)-\kappa[u(t, x) \\
& -u(t-T, x)],
\end{aligned}
$$

$$
\begin{aligned}
u_{x}(t, 0) & =u_{x}^{\text {desi }}(t, 0) & & \text { for } t>0, \\
u_{x}(t, L) & =u_{x}^{\text {desi }}(t, L) & & \text { for } t>0, \\
u(t, x) & =u_{0}(t, x) & & \text { for }(t, x) \in(-T, 0) \times(0, L) .
\end{aligned}
$$

While the above form of the equations nicely shows the action of Pyragas feedback stabilization, the following re-written partial differential equation fits better to the discussion of its analysis:

$$
u_{t}-u_{x x}+\rho R(u)+\kappa u=u_{t}(\cdot-T, \cdot)-u_{x x}(\cdot-T, \cdot)+\rho R(u(\cdot-T, \cdot))+\kappa u(\cdot-T, \cdot) .
$$

\subsection{Existence and Uniqueness of the Solutions}

Theorem 3 Suppose that $\rho \geq 0, u_{1} \leq u_{2} \leq u_{3}$, and $u_{0} \in L^{\infty}((-T, 0) \times(0, L))$ are given, such that the (classical) partial derivatives $\left(u_{0}\right)_{t},\left(u_{0}\right)_{x x}$ exist a.e. in $(-T, 0) \times(0, L)$ and

$$
\left(u_{0}\right)_{t}-\left(u_{0}\right)_{x x}+\rho R\left(u_{0}\right)+\kappa u_{0} \in L^{2}((-T, 0) \times(0, L)) .
$$

Suppose further that $u^{\text {desi }}$ obeys the properties assumed above. Then for all $T^{\prime} \geq T$ the parabolic initial-boundary value problem with time delay

$$
\begin{aligned}
u_{t}-u_{x x}+\rho R(u)+\kappa u= & u_{t}(\cdot-T, \cdot)-u_{x x}(\cdot-T, \cdot) & & \\
& +\rho R(u(\cdot-T, \cdot))+\kappa u(\cdot-T, \cdot) & & \text { in } Q_{T^{\prime}}, \\
u_{x}(t, 0)= & u_{x}^{\text {desi }}(t, 0) & & \text { in }\left(0, T^{\prime}\right), \\
u_{x}(t, L)= & u_{x}^{\text {desi }}(t, L) & & \text { in }\left(0, T^{\prime}\right), \\
u(t, x)= & u_{0}(t, x) & & \text { in }[-T, 0] \times(0, L),
\end{aligned}
$$

has a unique solution $u$ such that

$$
\left.u\right|_{\left[0, T^{\prime}\right]} \in L^{\infty}\left(Q_{T^{\prime}}\right) \cap W\left(0, T^{\prime}\right) \cap C\left(\left(0, T^{\prime}\right] \times[0, L]\right) .
$$

If $u_{0}$ belongs to $C([-T, 0] \times[0, L])$, then $u$ is also continuous on $\left[0, T^{\prime}\right] \times[0, L]$. 
Proof Again, we apply the step method. In the interval $[0, T]$, the right-hand side of the parabolic equation is

$$
\left(u_{0}\right)_{t}(t-T, x)-\left(u_{0}\right)_{x x}(t-T, x)+\rho R\left(u_{0}(t-T, x)\right)+\kappa u_{0}(t-T, x)=: F(t, x) .
$$

Thanks to our assumption, $F$ belongs to $L^{2}\left(Q_{T}\right)$. Now Theorem 5.5 of [32] can be applied that yields existence and uniqueness of a solution $u \in W(0, T) \cap C((0, T] \times[0, L]) \cap$ $L^{\infty}\left(Q_{T}\right)$ of

$$
\begin{aligned}
u_{t}-u_{x x}+\rho R(u)+\kappa u & =F & & \text { in } Q_{T}, \\
u_{x}(t, 0) & =u_{x}^{\text {desi }}(t, 0) & & \text { in }(0, T), \\
u_{x}(t, L) & =u_{x}^{\text {desi }}(t, L) & & \text { in }(0, T), \\
u(0, x) & =u_{0}(0, x) & & \text { in }(0, L) .
\end{aligned}
$$

Next, we extend the solution to $\left[T, \min \left\{2 T, T^{\prime}\right\}\right]$. Here, the new system reads

$$
\begin{aligned}
u_{t}-u_{x x}+\rho R(u)+\kappa u & =F(\cdot-T, \cdot) & & \text { in }\left(T, \min \left\{2 T, T^{\prime}\right\}\right) \times(0, L), \\
u_{x}(t, 0) & =u_{x}^{\text {desi }}(t, 0) & & \text { in }\left(T, \min \left\{2 T, T^{\prime}\right\}\right), \\
u_{x}(t, L) & =u_{x}^{\text {desi }}(t, L) & & \text { in }\left(T, \min \left\{2 T, T^{\prime}\right\}\right), \\
u(T, x) & =u(T-0, x) & & \text { in }(0, L) .
\end{aligned}
$$

Clearly, $F(\cdot-T, \cdot)$ belongs to $L^{2}\left(\left(T, \min \left\{2 T, T^{\prime}\right\}\right) \times(0, L)\right)$. Again we obtain existence, uniqueness, and regularity of $u$ on $\left[T, \min \left\{2 T, T^{\prime}\right\}\right]$ as above. If $T^{\prime}>2 T$, then we repeat the same procedure with $2 T$ as new initial time. After finitely many steps, we arrive at the final time $T^{\prime}$.

\subsection{Exponential Decay with Distributed Pyragas Control}

Now the stabilization result for periodic orbits is given.

Theorem 4 (Exponential stability) Let $T>0$ and a $T$-periodic state $u^{\text {desi }} \in H^{2}\left(Q_{2 T}\right)$ be given. Let the assumptions of Theorem 3 hold. Define

$$
\mu=2 \kappa-2 \rho\left|m_{R}\right| .
$$

Assume that $\mu>0$. The function

$$
V(t)=\frac{1}{2} \int_{0}^{L}(u(t, x)-u(t-T, x))^{2} d x
$$

is a strict Lyapunov function for the system $\mathbf{( S )}$ in the sense that for $t \geq T$ it satisfies the inequality

$$
V(t) \leq \exp (-\mu(t-T)) V(T) .
$$

Moreover, there exists a $T$-periodic function $u^{*} \in L^{2}\left(Q_{2 T}\right)$ and a constant $P_{0}>0$ such that for all $t \geq 0$ we have $u^{*}(t, \cdot) \in L^{2}(0, L)$ and the inequality

$$
\int_{0}^{L}\left(u(t, x)-u^{*}(t, x)\right)^{2} d x \leq P_{0} \exp (-\mu t)
$$

is satisfied for all $t \geq T$.

Proof By Theorem 3 the state $u$ is continuous. Hence, $V$ is well-defined by (1). The definition of $V$ implies that $V(t) \geq 0$. The pde (16) implies that for all 
$t \geq T$ in the sense of the solution of the system (S) presented in Theorem 3 we have

$$
\begin{aligned}
& u_{t}(t, x)-u_{t}(t-T, x)-\left[u_{x x}(t, x)-u_{x x}(t-T, x)\right] \\
& =-\rho[R(u(t, x))-R(u(t-T, x))]-\kappa[u(t, x)-u(t-T, x)] .
\end{aligned}
$$

We multiply (18) by $[u(t, x)-u(t-T, x)]$ and integrate to obtain for $t_{0}>T$

$$
\begin{aligned}
& \int_{T}^{t_{0}} \int_{0}^{L}[u(t, x)-u(t-T, x)]\left[\left[u_{t}(t, x)-u_{t}(t-T, x)\right]-\left[u_{x x}(t, x)-u_{x x}(t-T, x)\right]\right] d x d t \\
& =\int_{T}^{t_{0}} \int_{0}^{L}-\rho[u(t, x)-u(t-T, x)](R(u(t, x))-R(u(t-T, x)))-\kappa[u(t, x)-u(t-T, x)]^{2} d x d t .
\end{aligned}
$$

For initial data $u_{0}$ in $H^{2}((-T, 0) \times(0, L))$, integration by parts yields

$$
\begin{aligned}
& \left.\int_{0}^{L} \frac{1}{2}[u(t, x)-u(t-T, x)]^{2}\right|_{t=T} ^{t_{0}} d x \\
& =\int_{T}^{t_{0}} \int_{0}^{L}-\left[u_{x}(t, x)-u_{x}(t-T, x)\right]^{2} d x d t \\
& \quad+\left.\int_{T}^{t_{0}}[u(t, x)-u(t-T, x)]\left[u_{x}(t, x)-u_{x}(t-T, x)\right]\right|_{x=0} ^{L} d t \\
& \quad+\int_{T}^{t_{0}} \int_{0}^{L}-\rho[u(t, x)-u(t-T, x)](R(u(t, x))-R(u(t-T, x)))-\kappa[u(t, x)-u(t-T, x)]^{2} d x d t .
\end{aligned}
$$

Due to (2), for all $v_{1}, v_{2} \in(-\infty, \infty)$ it holds (14). With the definition of $V$ and since due to the periodic boundary conditions the terms at $x=0$ and $x=L$ cancel we obtain

$$
\begin{aligned}
V\left(t_{0}\right)-V(T) \leq & \int_{T}^{t_{0}} \int_{0}^{L}-\left[u_{x}(t, x)-u_{x}(t-T, x)\right]^{2} d x d t \\
& +\int_{T}^{t_{0}} \int_{0}^{L}\left(-\rho m_{R}-\kappa\right)[u(t, x)-u(t-T, x)]^{2} d x d t .
\end{aligned}
$$

Thus we have

$$
V\left(t_{0}\right) \leq V(T)+\int_{T}^{t_{0}} \int_{0}^{L}-\mu \frac{1}{2}[u(t, x)-u(t-T, x)]^{2} d x d t .
$$

Since $\mu>0$ this implies $V\left(t_{0}\right) \leq V(T)$. In fact, we have $0 \leq V\left(t_{0}\right) \leq V(T)-$ $\int_{T}^{t_{0}} \mu V(\tau) d \tau$. Now, Gronwall's lemma implies the inequality

$$
V\left(t_{0}\right) \leq V(T) \exp \left(-\mu\left(t_{0}-T\right)\right)
$$

With a density argument, this implies (17). The last part of the assertion follows as in the proof of Theorem 2 .

Example 2 Let $T=2 \pi$ and $L=1$. Assume that

$$
u_{x}^{\text {desi }}(t, 0)=u_{x}^{\text {desi }}(t, L)=0 .
$$

For $(t, x) \in(-T, 0) \times(0, L)$, let

$$
u_{0}(t, x)=\sin (t)
$$


Then system $(\mathbf{S})$ is

$$
\begin{aligned}
u(t, x)= & \sin (t) \quad \text { for }(t, x) \in(-2 \pi, 0) \times(0, L), \\
u_{x}(t, 0)= & 0 \quad \text { for } t>0, \\
u_{x}(t, L)= & 0 \text { for } t>0, \\
u_{t}= & u_{x x}-u_{x x}(\cdot-T, \cdot)-\rho[R(u)-R(u(\cdot-T, \cdot))] \\
& +u_{t}(\cdot-T, \cdot)-\kappa[u-u(\cdot-T, \cdot)] \quad \text { for } t>0 .
\end{aligned}
$$

The solution of the pde in (19) is in fact independent of $x$, so for $t>0$ the pde reduces to the ordinary differential equation

$$
u_{t}=u_{t}(\cdot-2 \pi, \cdot)-\rho[R(u)-R(u(\cdot-2 \pi, \cdot))]-\kappa[u-u(\cdot-2 \pi, \cdot)] .
$$

Since $u(t, x)=\sin (t)$ is $2 \pi$-periodic, it satisfies the above ordinary differential equation. Thus it is the $2 \pi$-periodic solution of (19).

\section{Stabilization to a Desired State}

In order to complete the picture, we also consider non-Pyragas distributed feedback. The analysis is completely analogous to Section 3 . Let a desired state $u^{\text {desi }} \in C^{2}([0, \infty) \times$ $[0, L])$ be given. Let initial data $u_{0}$ in $C^{2}([0, L])$ be given. Consider the system

$$
\begin{aligned}
u_{t}-u_{x x}+\rho R(u)+\kappa u & =u_{t}^{\text {desi }}-u_{x x}^{\text {desi }}+\rho R\left(u^{\text {desi }}\right)+\kappa u^{\text {desi }} & & \text { for }(t, x) \in(0, \infty) \times(0, L), \\
u_{x}(t, 0) & =u_{x}^{\text {desi }}(t, 0) & & \text { for } t>0, \\
u_{x}(t, L) & =u_{x}^{\text {desi }}(t, L) & & \text { for } t>0, \\
u(0, x) & =u_{0}(x) & & \text { for } x \in(0, L) .
\end{aligned}
$$

For the semilinear system (V), existence results from [16] apply.

Theorem 5 (Exponential stability) Define

$$
\mu=2 \kappa-2 \rho\left|m_{R}\right| .
$$

Assume that $\mu>0$. The function

$$
V(t)=\frac{1}{2} \int_{0}^{L}\left(u(t, x)-u^{\text {desi }}(t, x)\right)^{2} d x
$$

is a strict Lyapunov function for the system $(\mathbf{V})$ in the sense that for $t \geq 0$ we have

$$
V(t) \leq \exp (-\mu t) V(0)
$$

Proof The pde in (V) implies that we have

$$
u_{t}-u_{t}^{\text {desi }}-\left[u_{x x}-u_{x x}^{\text {desi }}\right]=-\rho\left[R(u)-R\left(u^{\text {desi }}\right)\right]-\kappa\left[u-u^{\text {desi }}\right] .
$$

We multiply (21) by $\left[u(t, x)-u^{\text {desi }}(t, x)\right]$ and integrate to obtain for $t_{0}>0$

$$
\begin{aligned}
& \int_{0}^{t_{0}} \int_{0}^{L}\left[u(t, x)-u^{\text {desi }}(t, x)\right]\left[\left[u_{t}(t, x)-u_{t}^{\text {desi }}(t, x)\right]-\left[u_{x x}(t, x)-u_{x x}^{\text {desi }}(t, x)\right]\right] d x d t \\
& =\int_{0}^{t_{0}} \int_{0}^{L}-\rho\left[u(t, x)-u^{\text {desi }}(t, x)\right]\left(R(u(t, x))-R\left(u^{\text {desi }}(t, x)\right)\right)-\kappa\left[u(t, x)-u^{\text {desi }}(t, x)\right]^{2} d x d t .
\end{aligned}
$$


Integration by parts yields

$$
\begin{aligned}
& \left.\int_{0}^{L} \frac{1}{2}\left[u(t, x)-u^{\text {desi }}(t, x)\right]^{2}\right|_{t=0} ^{t_{0}} d x \\
& =\int_{0}^{t_{0}} \int_{0}^{L}-\left[u_{x}(t, x)-u_{x}^{\text {desi }}(t, x)\right]^{2} d x d t+\left.\int_{0}^{t_{0}}\left[u(t, x)-u^{\text {desi }}(t, x)\right]\left[u_{x}(t, x)-u_{x}^{\text {desi }}(t, x)\right]\right|_{x=0} ^{L} d t \\
& +\int_{0}^{t_{0}} \int_{0}^{L}-\rho\left[u(t, x)-u^{\text {desi }}(t, x)\right]\left(R(u(t, x))-R\left(u^{\text {desi }}(t, x)\right)\right)-\kappa\left[u(t, x)-u^{\text {desi }}(t, x)\right]^{2} d x d t .
\end{aligned}
$$

Due to (2), for all $v_{1}, v_{2} \in(-\infty, \infty)$ it holds (14). With the definition of $V$ and since due to the boundary conditions the terms at $x=0$ and $x=L$ cancel we obtain

$$
\begin{aligned}
V\left(t_{0}\right)-V(0) \leq & \int_{0}^{t_{0}} \int_{0}^{L}-\left[u_{x}(t, x)-u_{x}^{d e s i}(t, x)\right]^{2} d x d t \\
& +\int_{0}^{t_{0}} \int_{0}^{L}\left(-\rho m_{R}-\kappa\right)\left[u(t, x)-u^{d e s i}(t, x)\right]^{2} d x d t .
\end{aligned}
$$

Thus we have

$$
V\left(t_{0}\right) \leq V(0)+\int_{0}^{t_{0}} \int_{0}^{L}-\mu \frac{1}{2}\left[u(t, x)-u^{\text {desi }}(t, x)\right]^{2} d x d t .
$$

Since $\mu>0$ this implies $V(0) \leq V(T)$. In fact, we have $0 \leq V\left(t_{0}\right) \leq V(0)-\int_{0}^{t_{0}} \mu V(\tau) d \tau$. Now, Gronwall's lemma implies the inequality $V\left(t_{0}\right) \leq V(0) \exp \left(-\mu t_{0}\right)$. Thus we have shown (20).

\section{Numerical Experiments}

\subsection{Numerical Results for Distributed Pyragas Feedback}

For simplicity we concentrate here on ordinary differential equations. They are equivalent to the Schlögl model, where spatially constant initial data functions $u_{0}(x, t) \equiv u_{0}(t)$ are given. Then the solution $u$ of the Schlögl model satisfies $u_{x x}=0$ and it is spatially constant for all times. Moreover, it obeys the homogeneous Neumann boundary conditions.

The feedback strategies of the previous sections partially generated periodic functions that are in fact constant. This is not a surprise, because certain standard solutions of the Schlögl model without time delay are constant. In the next following examples, we investigate the stabilization of delay equations that exhibit periodic solutions. By different stabilization strategies, we are able to generate as time limit functions of a desired period. However, in contrast to the preceding part, we were not yet able to prove this periodic limit behavior. Therefore, this subsection has an experimental character.

We define

$$
\rho=1, u_{1}=0, u_{2}=0.25, u_{3}=1, \quad R(u)=\left(u-u_{1}\right)\left(u-u_{2}\right)\left(u-u_{3}\right) .
$$

For delay $s=1.240683838477202$ and weight $\omega=-1.766552137106608$, let $v$ : $[-s, \infty) \rightarrow \mathbb{R}$ be the solution of the nonlinear delay equation

$$
\begin{aligned}
v^{\prime}(t) & =-\rho R(v(t))+\omega v(t-s) & & \text { for } t \in[0, \infty), \\
v(t) & =1 & & \text { if } t \leq 0 .
\end{aligned}
$$

These special numbers $s$ and $\omega$ were determined such that the solution $v$ of the nonlinear delay ode (22) in the interval $[0,160]$ minimizes the $L^{2}(0,160)$-distance to the solution of 


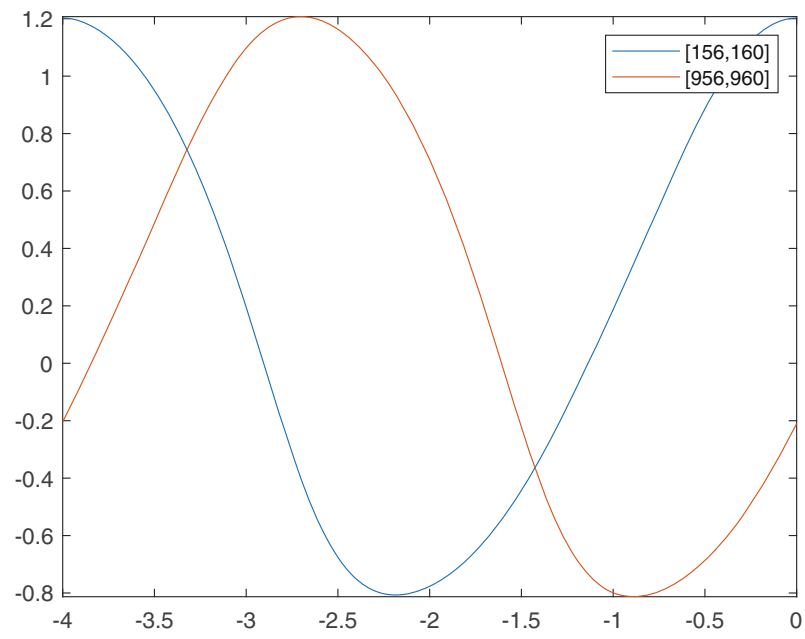

Fig. 1 The solution $v$ of the delay ode (22) does not seem to be 4-periodic

the linear delay ode

$$
\begin{aligned}
U^{\prime}(t) & =-\frac{\pi}{2} U(t-1) & & \text { for } t \in[0, \infty), \\
U(t) & =1 & & \text { if } t \leq 0 ;
\end{aligned}
$$

we refer to the computational examples in [7].

The function $U$ is known to be periodic with period $T=4$, cf. [13]. At first glance, the function $v$ seems to have this property, too. However, if we solve equation (22) in the interval $[0,960]$ and then compare the solution in $[156,160]$ with the solution in $[956,960]$, we can see that the obtained $v$ is not periodic of period 4; see Fig. 1. Most likely, some numerical instabilities are the reason for this behavior. On the other hand, the function $v$ might not be periodic.

Therefore, to obtain a periodic solution, we try to stabilize the solution of (22) by some feedback.

Strategy 1. We want to stabilize (22) using a Pyragas feedback stabilization. To obtain the target period $T=4$, we solve the equation

$$
\begin{aligned}
u^{\prime}(t) & =-\rho R(u(t))+\omega u(t-s)+\kappa(u(t-4)-u(t)) & & \text { for } t \in[160,960], \\
u(t) & =v(t) & & \text { if } 156 \leq t \leq 160
\end{aligned}
$$

with some positive $\kappa$. For a sufficiently large weight $\kappa, \kappa=100$ in our computations, we obtain a very fine numerical adjustment, cf. Fig. 2 . Indeed, the $L^{2}(-4,0)$ difference between $u(t+960)$ and $v(t+160)$ is $2 \times 10^{-2}$. Since $\|v\|_{L^{2}(156,160)}=1.4927$, we report only on absolute errors. In all the figures, the graph of the error is magnified 40 times with respect to the scale of the function.

Strategy 2. We try to stabilize the solution $v$ of (22) by adding the difference between the periodic target function

$$
u^{\text {desi }}=v(\bmod (t-160,4)+160-4)
$$




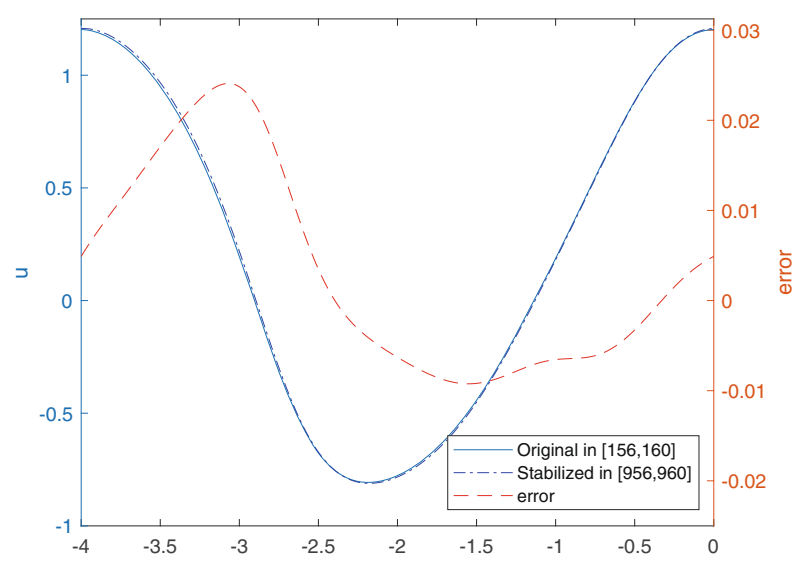

Fig. 2 The solution $u$ of the delay ode (23) with Pyragas feedback seems to be 4-periodic

and the solution $u$ of

$$
\begin{aligned}
u^{\prime}(t) & =-\rho R(u(t))+\omega u(t-s)+\kappa\left(u^{\text {desi }}(t)-u(t)\right) & & \text { for } t \in[160,960], \\
u(t) & =v(t) & & \text { if } 156 \leq t \leq 160 .
\end{aligned}
$$

For $\kappa=100$, the $L^{2}(-4,0)$-norm of the difference between $u(t+960)$ and $v(t+160)$ is $9 \times 10^{-3}$; see Fig. 3 .

Stabilization in the presence of small perturbations of parameters and data. The solution of (22) is very sensitive w.r.t. small perturbations of $s, \omega$ and the initial history $v(t)$ for $t \leq 0$. For instance, we consider the round off of $s$ and $\omega$ with two digits to the right of the decimal point,

$$
\hat{s}=1.24, \quad \hat{\omega}=-1.77 .
$$

Let $\hat{v}$ be the solution of the perturbed problem

$$
\begin{array}{cl}
\hat{v}^{\prime}(t)=-\rho R(\hat{v}(t))+\hat{\omega} \hat{v}(t-\hat{s}) & \text { for } t \in[0, \infty), \\
\hat{v}(t)=1 & \text { if } t \leq 0 .
\end{array}
$$

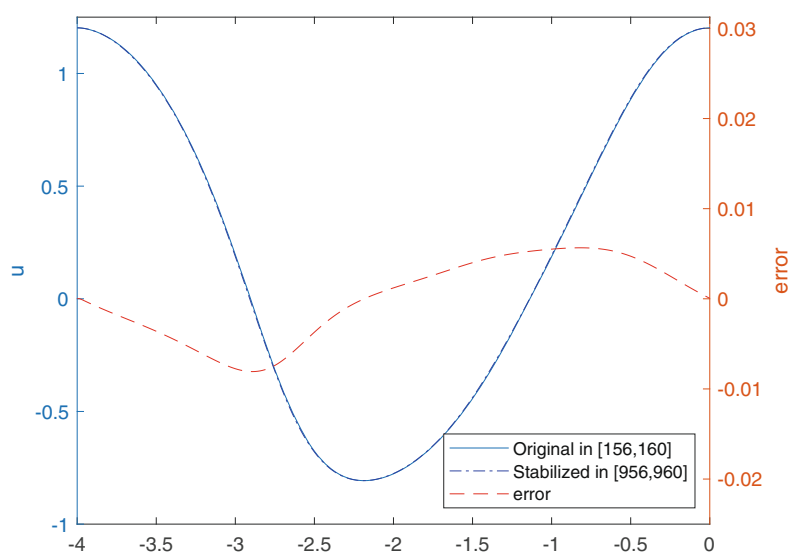

Fig. 3 The solution $u$ of the delay ode (24) with forcing term seems to be 4-periodic 


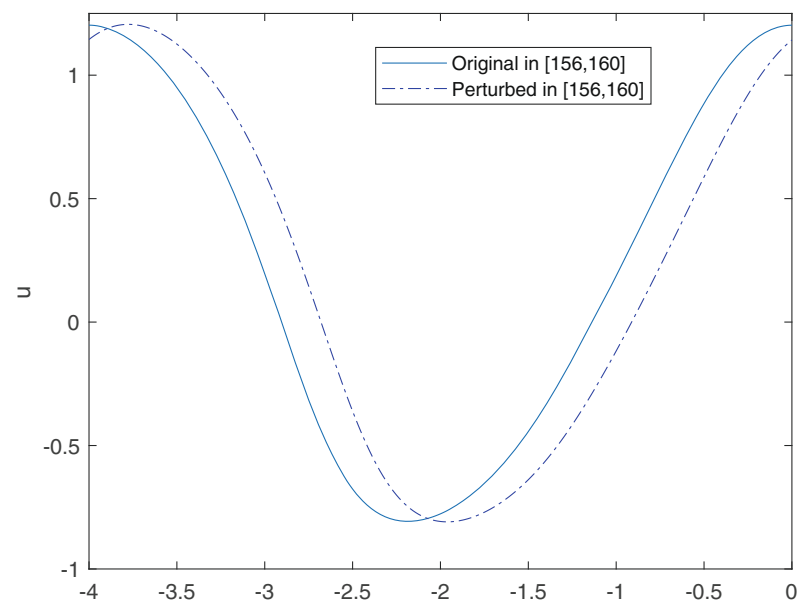

Fig. 4 The solution $\hat{v}$ of the perturbed delay equation (25) is quite different from the original solution $v$ of (22)

We obtain a solution that considerably differs from the original one in the last interval $[156,160]$. We have an error of $\|v-\hat{v}\|_{L^{2}(156,160)}=9 \times 10^{-1}$; see Fig. 4 .

Let us test the behavior of both stabilization strategies in this case.

Strategy 1 . We try to stabilize (25) using a Pyragas feedback stabilization. For the target period $T=4$, we solve the equation

$$
\begin{aligned}
u^{\prime}(t) & =-\rho R(u(t))+\hat{\omega} u(t-\hat{s})+\kappa(u(t-4)-u(t)) & & \text { for } t \in[160,960] \\
u(t) & =1.01 v(t) & & \text { if } 156 \leq t \leq 160
\end{aligned}
$$

for some positive $\kappa$. Notice that we have also perturbed the initial history. For $\kappa=100$, the $L^{2}(-4,0)$-norm of the difference between $u(t+960)$ and $v(t+160)$ is $1.5 \times 10^{-2}$. This is: the stabilized function not only appears to be periodic, but also is very close to the solution of the original unperturbed problem; cf. Fig. 5.

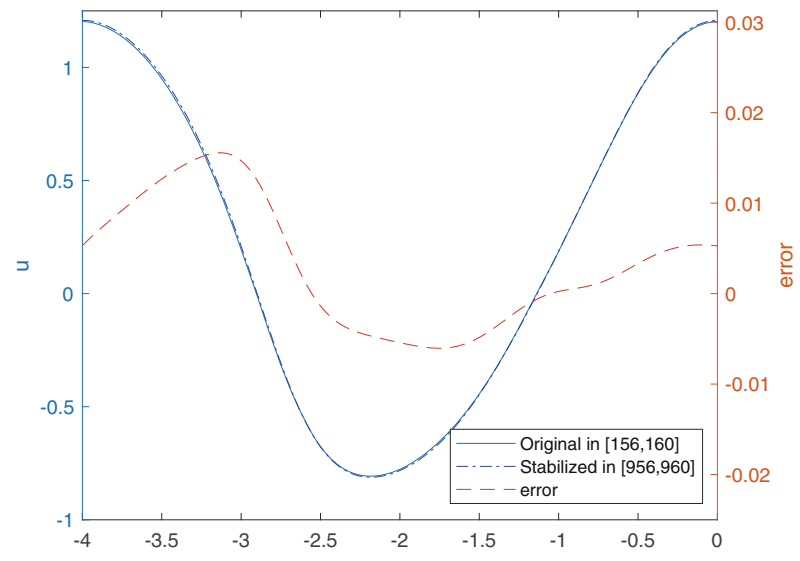

Fig. 5 The solution $u$ of the perturbed delay ode (26) with Pyragas feedback seems to be 4-periodic and is quite similar to the original solution $v$ of (22) 


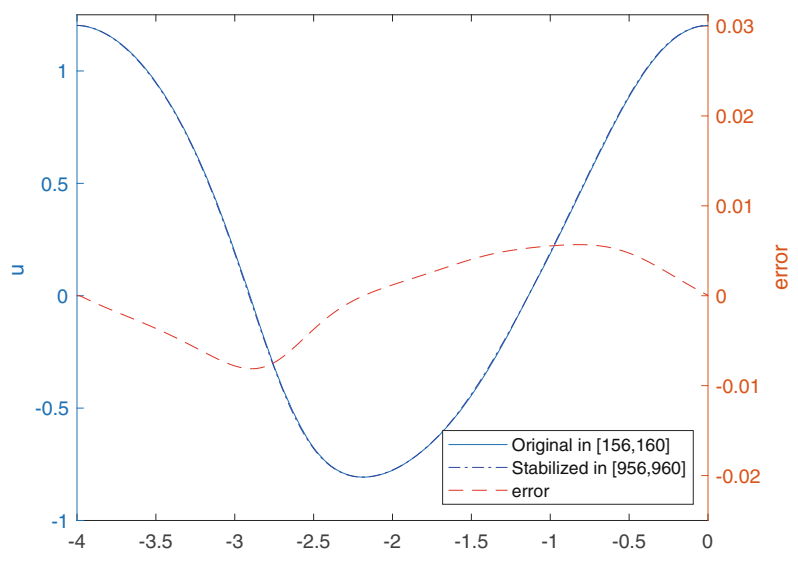

Fig. 6 The solution of the perturbed delay ode with forced feedback also seems to be 4-periodic and is quite similar to the original solution $v$ of (22)

Strategy 2. Using the target $u^{\text {desi }}(t)=v(\bmod (t-160,4)+160-4)$ instead of $u(t-4)$ yields also a successful stabilization. The error in this case is again $9 \times 10^{-3}$; cf. Fig. 6 .

Comment about perturbation. One could think that the periodic behavior is imposed only or mainly by the Pyragas feedback term because it has this big weight $\kappa$ and we impose a delay equal to the searched period. Nevertheless, if the ode we are trying to stabilize does not have a solution that is periodic (or is at least close to be periodic), then the Pyragas feedback term will not help to obtain such a periodic solution.

Our first motivation for this example was to mimic the solution of the linear delay equation (23), that has delay $\hat{s}=1$ and weight $\hat{\omega}=-\pi / 2$, with a the solution of the nonlinear ode (22). By an optimization method, we found the "strange" pair $s=1.240683838477202$

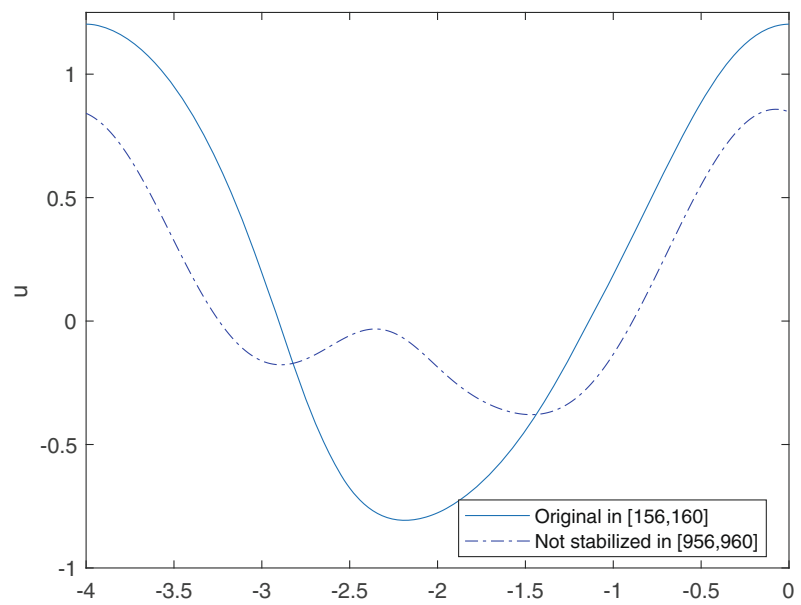

Fig. 7 The solution $u$ of the delay ode (27) cannot be steered to match the solution $v$ of the original delay ode (22) using a Pyragas feedback 


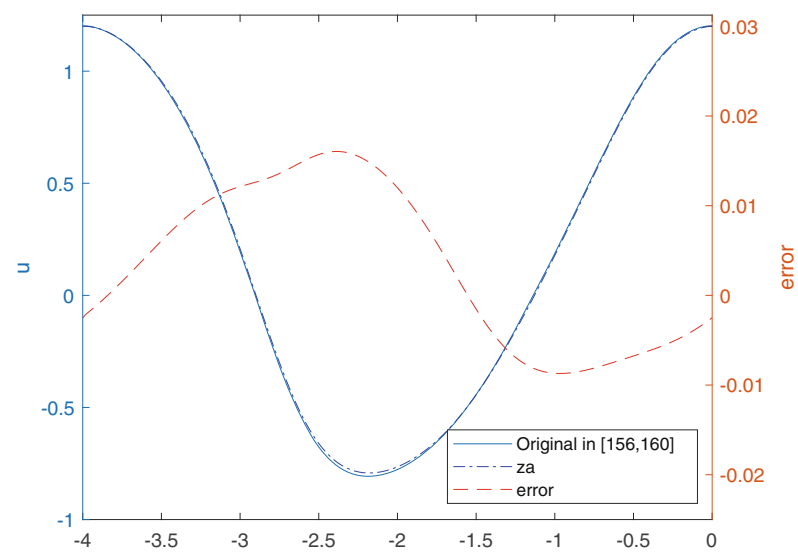

Fig. 8 The solution $u$ of an ode without delay, (28), is forced to match a periodic pattern by Strategy 2

and $\omega=-1.766552137106608$, cf. [7]. If we follow Strategy 1 and try to stabilize the perturbed equation inserting a Pyragas feedback term, i.e., solving the following equation,

$$
\begin{aligned}
u^{\prime}(t) & =-\rho R(u(t))-\frac{\pi}{2} u(t-1)+\kappa(u(t-4)-u(t)) & & \text { for } t \in[160,960] \\
u(t) & =v(t) & & \text { if } 156 \leq t \leq 160
\end{aligned}
$$

we are not successful; see Fig. 7.

However, Strategy 2 leads to a function that is very close to the solution $v$ of (22). In Fig. 8 we show, how the solution of

$$
\begin{aligned}
u^{\prime}(t) & =-\rho R(u(t))+\kappa\left(u^{\text {desi }}(t)-u(t)\right) & & \text { for } t \in[160,960] \\
u(t) & =v(t) & & \text { if } 156 \leq t \leq 160
\end{aligned}
$$

corresponding to $\omega=0$ (no delay) is lead to a periodic pattern by feedback Strategy 2 . The error in this case is $2 \times 10^{-2}$.

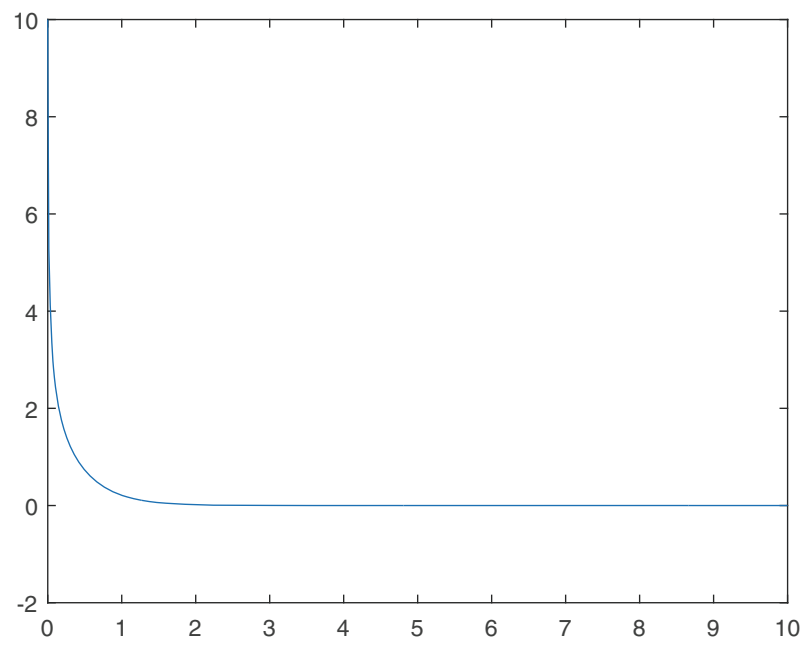

Fig. 9 The function $u(t)-\sin (t)$ for $\kappa=7$ 


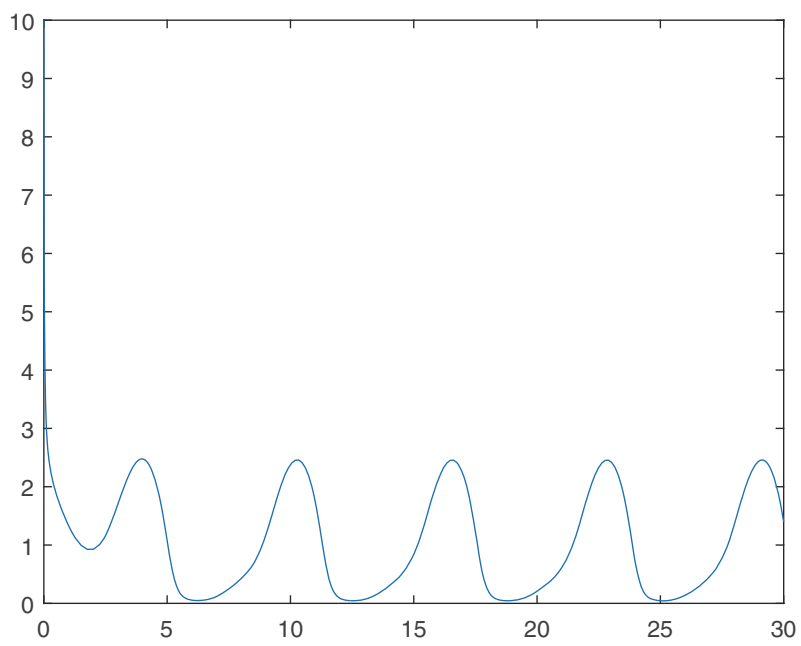

Fig. 10 The function $u(t)-\sin (t)$ for $\kappa=3$

\subsection{Numerical Results for Distributed (non-Pyragas) Feedback}

As in Example 1, we select the numbers $u_{1}=-1, u_{2}=0, u_{3}=\pi$. Then we have $R(u)=\left(u^{2}+u\right)(u-\pi)$ and also $m_{R}$ is as in Example 1. Let $\rho=1$ and $u^{\text {desi }}(t, x)=\sin (t)$. For constant initial data $u_{0}$, the solutions of system $(\mathbf{V})$ do not depend on $x$ and are the solutions of the Cauchy problem with the initial condition $u(0)=u_{0}$ and the ordinary differential equation

$$
u_{t}+\left(u^{2}+u\right)(u-\pi)+\kappa u=\cos (t)+\left(\sin ^{2}(t)+\sin (t)\right)(\sin (t)-\pi)+\kappa \sin (t) .
$$

Let $\kappa=7$. Since $\kappa>\rho\left|m_{R}\right|$, Theorem 4 implies that for any constant initial state the function $t \mapsto(u(t)-\sin (t))^{2}$ decays exponentially. This is illustrated by Fig. 9. The initial value for $t=0$ is $u_{0}=10$.

Numerical simulations indicate that for $\kappa=3$, the function $(u(t)-\sin (t))^{2}$ does not converge to zero for $t \rightarrow \infty$. This is illustrated by Fig. 10. Note however that the function $u(t)-\sin (t)$ appears to be periodic.

\section{Conclusion}

In this paper, feedback laws with Pyragas terms have been discussed that stabilize the Schlögl system globally to a $T$-periodic state for a given period $T>0$ under appropriate assumptions.

Both the case of Pyragas boundary feedback control on both ends of the interval and of distributed Pyragas feedback control were considered. A strict Lyapunov function was constructed to show the exponential stability of the resulting closed-loop system in the $L^{2}$ sense. We have presented inequalities that guarantee the exponential stability of the systems and can easily be verified. 
Acknowledgements Open Access funding provided by Projekt DEAL. This work was supported by DFG in the framework of the Collaborative Research Center Transregio 154, project C03. The second author was partially supported by Spanish Ministerio de Economía y Competitividad under research project MTM201783185-P.

Open Access This article is licensed under a Creative Commons Attribution 4.0 International License, which permits use, sharing, adaptation, distribution and reproduction in any medium or format, as long as you give appropriate credit to the original author(s) and the source, provide a link to the Creative Commons licence, and indicate if changes were made. The images or other third party material in this article are included in the article's Creative Commons licence, unless indicated otherwise in a credit line to the material. If material is not included in the article's Creative Commons licence and your intended use is not permitted by statutory regulation or exceeds the permitted use, you will need to obtain permission directly from the copyright holder. To view a copy of this licence, visit http://creativecommons.org/licenses/by/4.0/.

\section{References}

1. Alasty, A., Salarieh, H.: Nonlinear feedback control of chaotic pendulum in presence of saturation effect. Chaos Solut. Fractals 31, 292-304 (2007)

2. Barbu, V., Cordoni, F., Di Persio, L.: Optimal control of stochastic FitzHugh-Nagumo equation. Int. J. Control 89, 746-756 (2016)

3. Breiten, T., Kunisch, K.: Feedback stabilization of the Schlögl model by LQG-balanced truncation. In: 2015 European Control Conference (ECC), pp. 1171-1176. IEEE (2015)

4. Brouwer, J., Gasser, I., Herty, M.: Gas pipeline models revisited: model hierarchies, nonisothermal models, and simulations of networks. Multiscale Model. Simul. 9, 601-623 (2011)

5. Buchholz, R., Engel, H., Kammann, E., Tröltzsch, F.: On the optimal control of the schlögl-model. Comput. Optim. Appl. 56, 153-185 (2013)

6. Casas, E., Ryll, C., Tröltzsch, F.: Sparse optimal control of the schlögl and FitzHugh-Nagumo systems. Comput. Methods Appl. Math. 13, 415-442 (2013)

7. Casas, E., Mateos, M., Tröltzsch, F.: Optimal time delays in a class of reaction-diffusion equations. Optimization 68, 255-278 (2019)

8. Chen, Z.-X., Guo, B.-Y.: Analytic solutions of the Nagumo equation. IMA J. Appl. Math. 48, 107-115 (1992)

9. Cordoni, F., Di Persio, L.: Optimal control for the stochastic FitzHugh-Nagumo model with recovery variable. Evol. Equ. Control Theory 7, 571-585 (2018)

10. Coron, J.-M., d'Andréa-Novel, B., Bastin, G.: A strict Lyapunov function for boundary control of hyperbolic systems of conservation laws. IEEE Trans. Autom. Control 52, 2-11 (2007)

11. Coron, J.-M.: Control and Nonlinearity. American Mathematical Society, Providence (2007)

12. Domschke, P., Hiller, B., Lang, J., Tischendorf, C.: Modellierung von Gasnetzwerken: Eine Übersicht. Preprint, TRR 154 (2017)

13. Erneux, T.: Applied Delay Differential Equations. Surveys and Tutorials in the Applied Mathematical Sciences, vol. 3. Springer, New York (2009)

14. Gopalakrishnan, A., Biegler, L.T.: Economic nonlinear model predictive control for periodic optimal operation of gas pipeline networks. Comput. Chem. Eng. 52, 90-99 (2013)

15. Grassberger, P.: On phase transitions in schlögl's second model. Z. Phys. B Condens. Matter 47, 365-374 (1982)

16. Gugat, M., Tröltzsch, F.: Boundary feedback stabilization of the schlögl system. Automatica 51, 192-199 (2015)

17. Holt, A.B., Netoff, T.I.: Origins and suppression of oscillations in a computational model of Parkinson's disease. J. Comput. Neurosci. 37, 505-521 (2014)

18. Karzari, M.A., Lemarchand, A., Mareschal, M.: Fluctuation effects on chemical wave fronts. Phys. Rev. E 54, 4888-4895 (1996)

19. Kunisch, K., Wagner, M.: Optimal control of the bidomain system (III): Existence of minimizers and first-order optimality conditions. ESAIM Math. Model. Numer. Anal. 47, 1077-1106 (2013)

20. Lehnert, J., Hövel, P., Selivanov, A., Fradkov, A., Schöll, E.: Controlling cluster synchronization by adapting the topology. Phys. Rev. E 90, 042914 (2014)

21. Lunardi, A.: Analytic Semigroups and Optimal Regularity in Parabolic Problems. Progress in Nonlinear Differential Equations and Their Applications, vol. 16. Basel, Birkhäuser (1995) 
22. Mazenc, F., Prieur, C.: Strict Lyapunov functions for semilinear parabolic partial differential equations. Math. Control Relat. Fields 1, 231-250 (2011)

23. Merat, K., Chekan, J.A., Salarieh, H., Alasty, A.: Control of discrete time chaotic systems via combination of linear and nonlinear dynamic programming. J. Comput. Nonlinear Dyn. 10, 011008 (2015)

24. Morgül, Ö.: A new generalization of delayed feedback control. Int. J. Bifurc. Chaos 19, 365-377 (2009)

25. Nagumo, J., Arimoto, S., Yoshizawa, S.: An active pulse transmission line simulating nerve axon. Proc. IRE 50, 2061-2071 (1962)

26. Newell, A.C., Whitehead, J.A.: Finite bandwidth, finite amplitude convection. J. Fluid Mech. 38, 279303 (1969)

27. Nieto, J.J.: Periodic solutions of nonlinear parabolic equations. J. Differ. Equ. 60, 90-102 (1985)

28. Schlögl, F.: Chemical reaction models for non-equilibrium phase transitions. Z. Phys. A 253, 147-161 (1972)

29. Segel, L.A.: Distant side-walls cause slow amplitude modulation of cellular convection. J. Fluid Mech. 38, 203-224 (1969)

30. Selivanov, A., Lehnert, J., Fradkov, A., Schöll, E.: Adaptive time-delayed stabilization of steady states and periodic orbits. Phys. Rev. E. 91, 012906 (2015)

31. Stolwijk, J.J., Mehrmann, V.: Error analysis and model adaptivity for flows in gas networks. Anal. Univ. "Ovidius" Constanta Ser. Mat. 26, 231-266 (2018)

32. Tröltzsch, F.: Optimal Control of Partial Differential Equations: Theory, Methods and Applications. Graduate Studies in Mathematics, vol. 112. AMS, Providence (2010)

Publisher's Note Springer Nature remains neutral with regard to jurisdictional claims in published maps and institutional affiliations. 